\title{
The Effects of Personal Displays and Transfer Techniques on Collaboration Strategies in Multi-touch Based Multi-Display Environments
}

\author{
Stefan Bachl ${ }^{1}$, Martin Tomitsch ${ }^{2}$, Karin Kappel ${ }^{1}$, and Thomas Grechenig ${ }^{1}$ \\ ${ }^{1}$ Research Group for Industrial Software (INSO), Vienna University of Technology, \\ Wiedner Hauptstr. 76/2/2, 1040 Wien, Austria \\ \{stefan.bachl, karin.kappel, thomas.grechenig\} @inso.tuwien.ac.at \\ ${ }^{2}$ Design Lab - Faculty of Architecture, Design and Planning, The University of Sydney, \\ 148 City Road, NSW 2006, Sydney, Australia \\ martin.tomitschesydney.edu.au
}

\begin{abstract}
Multi-touch tabletop systems promise to enhance collaboration in multi-display (MDE) environments. However, little is known about the effects of combining shared multi-touch tabletops with multi-touch tablet computers (tablets) as the collaborators' personal displays. In this paper we present the implementation of a MDE with multi-touch input on both shared and personal displays and its evaluation regarding task performance, user preferences and collaboration strategies compared to a standard multi-touch tabletop setting. Eight participant pairs had to solve a collaborative sorting task using three different transfer techniques. Based on an analysis of video recordings, log files and user feedback we identified task solving and collaboration strategies. The use of tablets enabled participants to follow diverse strategies and participants preferred the collaboration using tablets, while overall task performance and the amount of close collaboration were higher without the tablets.
\end{abstract}

Keywords: Co-located collaboration, interactive surfaces, multi-display environment, multi-touch, tabletop interaction.

\section{Introduction}

In the last few years multi-touch surfaces have gained a lot of attention, not only because of the increasing presence in consumer products and advances in finger tracking technologies but also because of benefits that come with this form of interaction. Existing challenges regarding the interaction design of multi-touch interfaces are balanced by advantages that come with this technology such as intuitive, direct interaction $[3,12]$, gestures [33] and the possibility of concurrent colocated collaboration. Multi-touch surfaces promise to enhance collaboration and to allow creating more engaging, flexible and easy to use interfaces to support the activities and workflows of teams working together [6,9].

Additionally, portable devices are becoming more flexible and easy to use. Mobile phones and tablet computers continue to increase in functionality. Paired with instant 
on ability, portable devices are slowly finding their way into collaborative multidisplay environments [2, 19]. For instance, a collaborative environment can support the integration of mobile devices into a shared network [25], the transfer and sharing of documents between devices to facilitate collaboration, or other more complex collaborative workspace scenarios [4, 20, 26, 27]. Consequently, synchronization and transfer techniques for sharing documents between devices become more important, whether data is exchanged locally or via the cloud. Emerging technologies even encourage approaches for transferring documents through digital representations projected onto users' hands while moving through a room [32].

Most collaborative environments have one thing in common: They are based on a shared space where collaborators can interact and communicate and coordinate their tasks. In digitally supported collaborative environments, this shared space is often represented by multi-touch tabletop systems. There is a large body of research available that investigated interactions with and around multi-touch tabletops and their effects on collaboration and task performance [8, 10, 15, 23, 24]. However, little information is available regarding techniques for sharing and transferring digital content between several multi-touch surfaces and the role of personal devices in digitally supported collaborative environments.

In this study we therefore investigate a multi-display environment consisting of a shared tabletop display and personal devices with multi-touch support on all devices. More specifically, we investigated the effects of personal displays and transfer technique on performance, awareness and collaboration strategies. For this purpose we propose three unidirectional transfer techniques for sending documents from the shared surface to personal displays, represented by tablet computers.

\section{Related Work}

Related work in the area of collaborative tabletop environments can be separated into single- and multi-display environments. Single-display environments (SDE) include all setups with a shared display for collaboration and either no additional input or one additional input device for each participant. Examples for input devices are computer mice or pen-based graphical tablet computers. Multi-display environments (MDE) consist of one or multiple shared displays for collaboration and include additional input devices with displays for each collaborator. Examples for input devices with displays are mobile phones, laptop computers, pen-based tablet computers (tablets), or as in our study (multi-)touch tablets.

\subsection{Single-Display Environments}

Hornecker et al. [9] compare a multi-touch tabletop setup where collaborators can interact with their fingers to a setup where instead each participant controls the interface with a computer mouse. Measuring the awareness of participants about collaborators' activities and their overall shared understanding of behavior, they conclude that the multi-touch tabletop system results in higher awareness and consequently to better task performance and results. Müller-Tomfelde and Schremmer [14] compare multi-touch with mouse interaction on a shared surface by allowing 
individual participants to choose their preferred input method. Although the input does not have an impact on performance, the authors state that participants are likely to stick to their initial input preferences. Isenberg et al. [10] identify different collaboration styles of participants working on a collaborative problem-solving task, analyze their strategies and conclude with design implications. Rogers and Lindley [22] compare a horizontal to a vertical display with pen-based input on both. They conclude that the vertical setup is better for communal and audience-based viewing and annotating, whereas the horizontal setup is superior for collaborative activities.

\subsection{Multi-Display Environments}

Wallace et al. [31] use a collaborative setup with a vertical display (acting as output only) to compare input with multiple mice to laptop computers. They conclude that collaborators using computer mice have a higher awareness of their partners' activities. Collaborators break off to individual tasks rather than monitor the shared activities when working with a laptop computer. Hawkey et al. [8] use a pen-based digital whiteboard system in combination with pen-based tablet computers to investigate the effects of distance between collaborators and the digital whiteboard on interaction and awareness. They conclude that the collaboration intensifies when users are near to the whiteboard system and also close to each other. Shen et al. [25] examine an interactive multi-touch tabletop for face-to-face collaboration where users can easily share their content from personal devices such as laptop computers. They identify three collaboration areas: The private area is not visible or accessible for others, the personal area is visible, but not accessible for others and the public (or shared) area is visible and accessible for collaborators. Nacenta et al. [17] use a penbased horizontal surface with stationary pen-based tablets to investigate different transfer techniques between both devices together. The transfer techniques are tested with different distances between the stationary tablet and the horizontal surface. The authors describe design directions and recommendations for the use of individual transfer techniques. Tan et al. [28] present a collaborative job-shop scheduling task for MDEs with mouse-based interaction only. Nacenta et al. [16] present factors in MDEs and cross-display object movement techniques.

\section{Supporting Transfer Techniques in Multi-Display Environments}

We propose three one-way transfer techniques for sharing digital documents between a shared and personal multi-touch displays in a collaborative MDE. We developed the three techniques together with the interfaces for both shared and personal displays.

\subsection{Shared Display}

Each document is represented by a virtual representation on the shared display showing the entire content of the document. Users can resize documents within a predefined scale range and move them across the surface. The system allows users to group documents regardless of size (Figure 1) by moving two documents' side edges close to each other and releasing the documents. Grouped documents can be moved as 
a unit and individual documents can still be resized. To separate grouped documents users have to touch two adjacent documents, start dragging them apart from each other and release them. For instance, this grouping interaction can support sorting of a large number of individual documents.

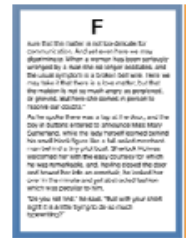

(1)

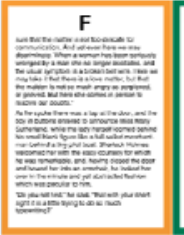

(2)

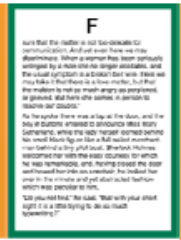

(3)

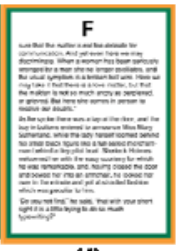

(4)

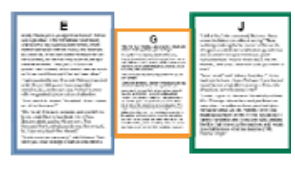

(6)

Fig. 1. Documents on the shared display are represented as single-page representations with color-coding indicating documents that are currently opened by any of the collaborators on their tablets (1-4). Documents can be attached to each other along their vertical edges and single documents remain resizable (5).

\subsection{Personal Display}

The personal display features a document tray at the bottom of the screen where small representations of documents that are currently opened on the tablet are displayed. Touching a document's representation opens the respective contents on the document view area and highlights the representation in the tray. Each document's representation contains a small circular button at the top right corner to close the document. The brightness of the document view area is reduced to provide comfortable reading conditions. A document opened on a tablet also keeps its representation visible on the shared surface. We use color-coding for identifying users in the shared environment. When a user opens a document on their tablet, a border with the corresponding color is displayed around the document's representation on the shared display. The same color-coding is used on the tablets to support the mapping between tablet and user (Figure 1). If two or more users open a document at the same time, multiple borders with the respective colors are added to the document's representation on the shared display (Figure 1). This visualization helps users to easily identify and track opened documents. For simplicity reasons users can open only up to five documents at the same time on each tablet. When users try to open more than five documents at a time, an according message is displayed in form of a pop-up on the tablet. Users can resize a document's representation on the shared screen and scroll longer documents through simple touch interaction on the tablet. Participants are not able to change the order of documents or attach documents to each other on the tablets.

\subsection{Transfer Techniques}

Based on our review of related work we identified five techniques for supporting document transfer in MDEs through continuous touch interaction. Mouse and pen input were disregarded to avoid any negative effects of changing the mode of 
interaction between devices and since their differences to multi-touch were already examined by others [3, 9, 14, 18]. For further selection we evaluated the five identified techniques in a preliminary user study. Two techniques, a variation of pickand-drop [21] and a three-finger multi-touch swipe gesture, were dropped due to limitations identified in this preliminary study. More specifically, we observed that in our context multi-touch gestures did not have any benefits for document transfer and were less accepted by users.

The final transfer techniques described below represent three distinct investigations into the design space allowing users to transfer documents from the shared display onto their personal displays through directly selecting the document on the shared display (buttons), moving the document onto a virtual representation of the personal display (containers), or selecting the document on the personal display (lenses). The proposed system only supports unidirectional transfer of documents from the shared display to the users' personal displays but not vice versa (Figure 2).
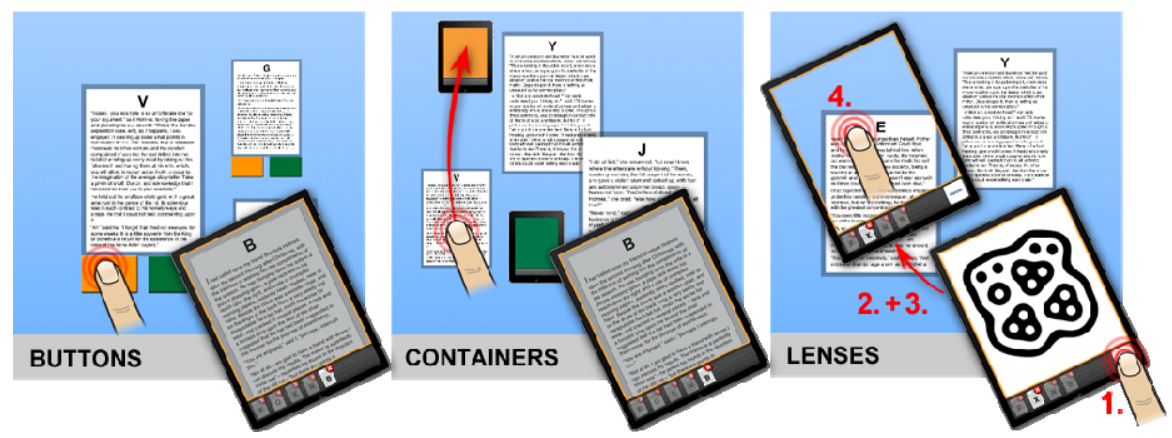

Fig. 2. Schematic representations showing the three different transfer techniques. Users can touch buttons to transfer an individual document (left), drag-and-drop documents or groups of documents into containers (middle) or use the tablets as lenses by activating the lens mode (1), selecting an area showing the desired document(s) (2), transferring the area onto the tablet (3), and selecting document(s) to open on the tablet (4).

Buttons. To support the buttons transfer technique, each document on the shared display features one button per user attached to an edge of the document's representation. The buttons are associated with users through color mapping. Touching a button (Figure 2) transfers the document onto the corresponding tablet. The buttons technique enables users to send documents to their collaborators.

Containers. In the containers transfer technique a visual element on the shared surface represents a user's tablet. The number of visual elements corresponds to the number of tablets connected to the system. We use an icon representing a tablet as visual element to convey its meaning as container mapped to the user's tablet. Again, we use color for mapping containers with tablets. Users can move the containers across the screen to allow positioning for convenient access. Containers are not resizable to avoid confusion with the document elements and always remain on top to avoid occlusion. Employing the drag-and-drop metaphor, users can drag a single document or groups of attached documents and drop them into one of the containers 
(Figure 2), which consequently opens them on the corresponding tablet. Documents remain on the shared display and jump back to their initial position of the drag gesture after the transfer has been completed. Like the buttons technique, the containers technique also enables users to send documents to their collaborators.

Lenses. In the lenses transfer technique tablets act as lenses and can capture parts of the content of the shared display. We use fiduciary markers for detecting the positions of the tablets (see section 4.5). The interface of the tablets features an additional button in the bottom right corner to activate the lens mode (Figure 3 ). While holding the button users can move the tablet above the shared surface. A translucent representation of the tablet is displayed on the shared surface to support this interaction and provide feedback. When releasing the button, the content of the tablet's viewing area changes to the exact view of the shared display that is displayed beneath the current position of the tablet (Figure 2 and Figure 3). Participants can open documents on their tablet by simply touching their representations captured through the lens. When touching the button in the bottom right corner again or tilting the tablet while in lens mode, users return to their previously opened document.
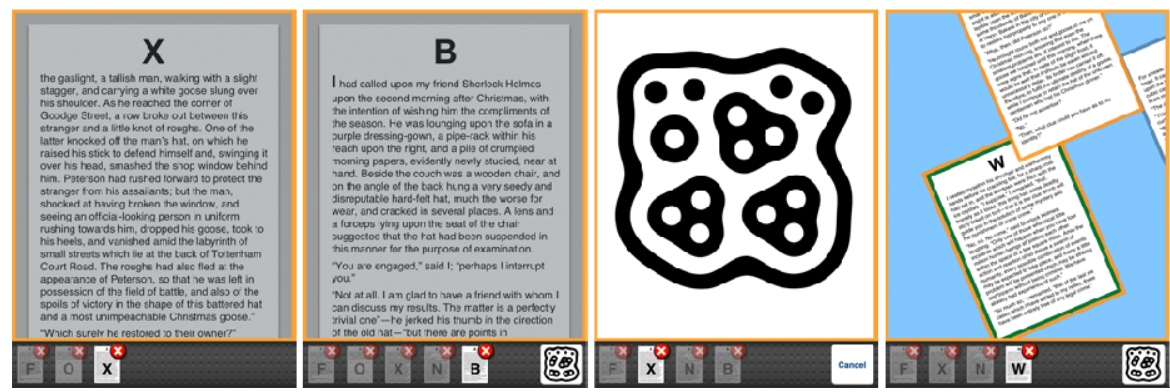

Fig. 3. Screenshots of the software running on the personal displays. Participants could read documents (first image) with reduced brightness. For the lenses transfer technique, an additional button was displayed in the bottom right corner (second image). When pressing the button a fiduciary marker was shown (third image). After releasing the contents of the shared surface were displayed (last image).

\section{Evaluation Study}

To investigate the proposed transfer techniques we conducted a task-based study with eight participant pairs. We used a within-subject design with four conditions in total, three being the different transfer techniques, and one allowing participants to only use the shared display to solve the given task. Conditions were counterbalanced to eliminate learning effects.

\subsection{Task}

We prepared a task, which required participant pairs to collaboratively sort ten pages of a book chapter. Ten single-page documents were randomly placed (all reduced to minimum size) on the shared display at the start of each condition. The goal was to 
bring the documents into the correct order by grouping pages together. Four chapters of "The Adventures of Sherlock Holmes" by Sir Arthur Conan Doyle were retrieved from Project Gutenberg [5]. The length of each chapter was reduced to about 2,100 words and separated into ten single pages. Some pages ended with full stops, others were separated in the middle of a sentence. The text also contained dialogs that helped participants to identify pages that belonged together. The first paragraph on the first page contained a large-sized capital letter to provide a starting point for solving the task. The text on the last page did not fill the whole page to help identifying the last page. These clues were given intentionally to enable participants to solve the task in a reasonable amount of time, as they had to complete the task for each condition. Participant pairs were presented with a different chapter from the book for each condition. At the top of each document, a randomized letter between $\mathrm{A}$ and $\mathrm{Z}$ in a large font was placed for identification. The letter helped participants to identify documents, but also to support communication (e.g. "I think ' $E$ ' goes before ' $F$ "'). The text size in the PDF-documents was set to 25 points to make it readable on both shared and personal displays, although on the shared display legibility depended on the scale factor applied to a document.

\subsection{Participants}

For our study we recruited 16 participants ( 9 male, 7 female), aged between 19 and 47 years (mean: 25.7; SD: 7.07), and grouped them into eight pairs to examine the "collaborative coupling style" between participant pairs [29, 30]. 13 of the 16 participants were students from the University of Sydney. In six of the eight pairs participants already knew their partner before taking part in the experiment. Participants were partners, co-workers or friends. Participants were screened before the experiment in order to make sure that their English skills were sufficient to solve the given task. Six participants were native English speakers. On a scale between "fluent", "good" and "fair" eight participants rated their English skills as "fluent" and only two participants as "fair". None of the participants had read "Sherlock Holmes" within the last 5 years. Two of the 16 participants were left-handed. Participants were also tested for color-blindness. Three of the 16 participants did not have experiences with touch interfaces, five participants had used large multi-touch displays before.

\subsection{Procedure}

Before beginning with the experiment participants gave their informed consent to the study. Participants were asked to sit or stand in front of the tabletop next to each other. A within-subject design was chosen: Each participant pair was asked to complete the task consecutively in all four conditions (with different book chapters). The first condition (without) only involved the shared display, the other three included the use of the tablet computers as personal displays. In the conditions with personal displays participants were able to transfer documents from the tabletop onto their tablets. Each condition supported one of the three specific techniques for transferring documents (Figure 4). 


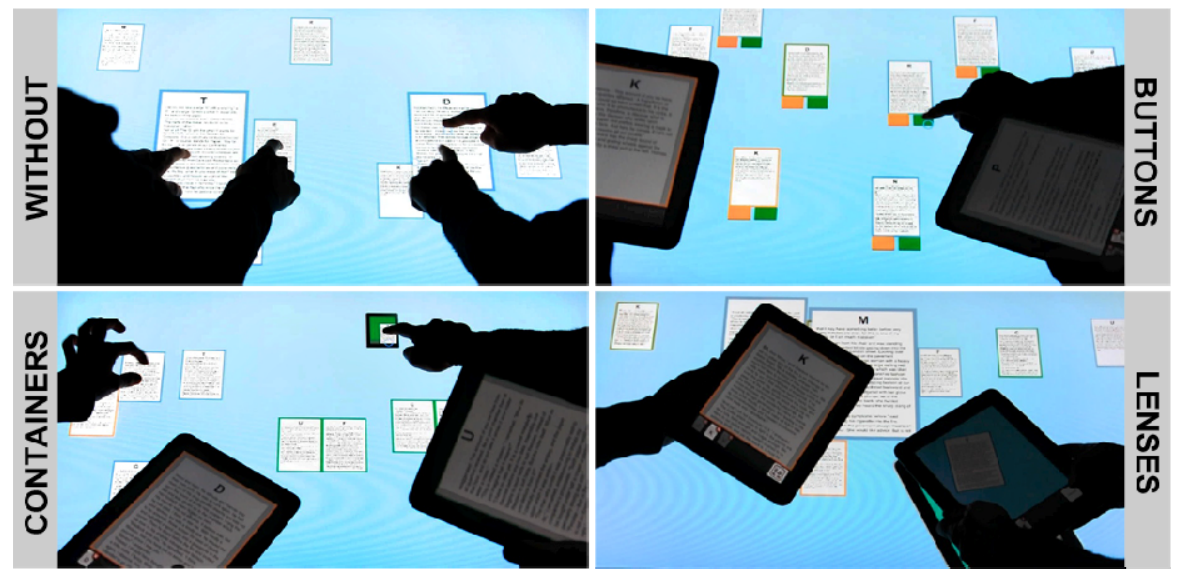

Fig. 4. Frames of a video showing the four conditions of our study: Working without tablets (top left), using buttons to transfer documents to the tablets (top right), drag-and-dropping documents into containers (bottom left) and using the tablets as lenses displaying parts of the contents of the shared surface (bottom right)

To eliminate potential learning effects within the groups, the order of the conditions was counterbalanced using Latin Squares. A short tutorial was given to the participants prior to each condition. During the tutorial participants had the opportunity to briefly interact with the system to make sure they understood the interface and transfer technique (in the conditions that involved a tablet). After the introduction the system was restarted and set up for the experiment. This procedure ensured that all participants understood the task and the transfer techniques. Except for a few questions during the experiments, additional help or assistance was not required. The task completion time per condition was about 11.5 minutes on average. Participants had the option to take a break after the first two conditions. After completing all four conditions, participants were asked to complete a questionnaire. Additionally, a short interview consisting of open questions with both participants together was conducted to gain deeper insights into their personal preferences and observations.

\subsection{System Implementation}

The system used for the evaluation consisted of two parts, a software running on the main computer controlling the shared surface and a separate piece of software for controlling the personal tablet computers. The system allowed documents displayed on the shared display to be transferred onto the tablets using the transfer techniques described above (Figure 4).

The application running on the main computer controlled the shared display and communicated with the tablet computers. The Java-based multi-touch framework MT4J [13] was used for implementation. The main application processed messages 
received from the tracking software. The communication between the tracking software and our application was necessary to identify the positions of the users' fingers touching the surface and also the positions of the fiduciary markers in order to identify the positions of the tablets used in the lenses transfer technique. The rotation of documents on the shared display was disabled, as it would have added complexity for the action of grouping documents.

The application running on the tablet computer was implemented with the Cocoa Touch framework (native iOS development based on Objective-C) since first generation Apple iPads are used for the underlying hardware platform. To enable fast switching of variables, experiment settings (e.g. condition or participant identification) were included in the tablet's global settings outside of the application. The tablet computers were connected with the main application via a wireless network connection to communicate with each other. The PDF documents were redundantly stored on the main computer and both tablets. To minimize latency just the corresponding identification numbers to open the documents were synchronized instead of transferring the document files themselves.

\subsection{Experimental Setup}

The study was conducted in one of our faculty's lab spaces. A custom-made horizontal multi-touch display built into a table was used as the shared display. The 42-inch display with a resolution of 1600x900 pixels was augmented with Laser Light Plane Illumination (LLP) to track the touches of participants' fingers. Two cameras were used to track interactions: One infrared camera was connected to a computer running Community Core Vision (CCV) [1], tracking the touches of the participants' fingers. A second visible light camera was connected to another computer running ReacTIVision [11] to track the fiduciary markers in the lenses condition. Even though ReacTIVision is capable of tracking both fingers and fiduciary markers, we chose to use CCV for tracking finger interactions because of its higher accuracy. The main application for the shared display was running on the second computer. All computers including the two tablets were connected via a wireless network connection. No performance issues emerged during the experiments.

Two first generation Apple iPads were used as the personal displays. Their 9.7inch capacitive multi-touch display featured a resolution of $1024 \times 768$ pixels. Compared to the shared display, the iPads were slightly superior in regards to accuracy and display resolution.

A video camera was mounted at the side of the tabletop to record interactions of the participants on the shared display. For privacy reasons the faces of the participants were not captured. In all four conditions, chairs were provided for sitting at the table, but participants were explicitly told that they could decide on their own whether they preferred to stand or sit while completing the task. The cameras for tracking the interactions were mounted at one side of the table. Therefore, participants could only work on the opposite side. The tabletop featured additional horizontal space on its shorter sides, which participants could use to put down the tablets. 


\subsection{Data Collection and Analysis}

To ensure the quality of the data collected during the experiment, we used several sources for recording data. The video camera recorded interactions on the shared display. The main application controlling the shared display and the two applications running on the tablet computers logged all user interactions and stored them in a database. Additionally, we used data retrieved from the questionnaires, interviews and logging sheets for our analysis.

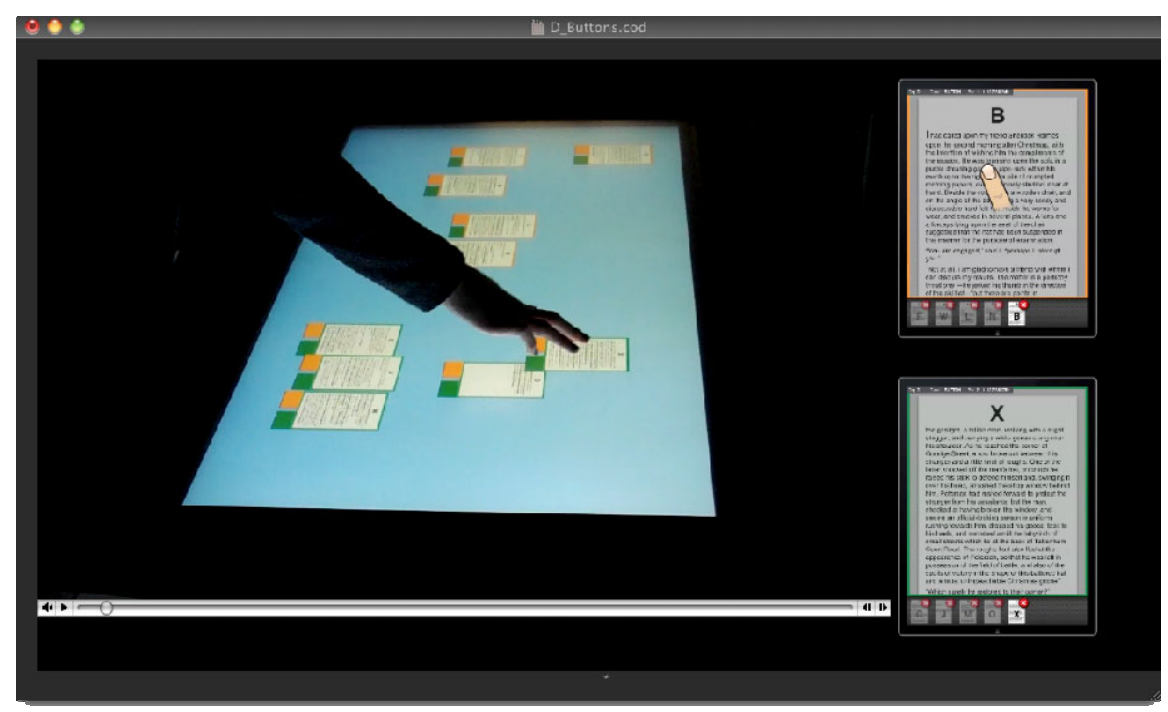

Fig. 5. A screenshot showing a reduced view of the video coding tool VCode [7]. Video recordings of interactions during the experiment on the shared surface (left) were combined with log file-based visual simulations of interactions on the two tablets (right).

For the video analysis we used VCode [7] and adapted the controls of the video playback with custom keyboard shortcuts for better control. Our video footage did not contain interactions on the tablets. Therefore we modified the original application running on the tablets to simulate (play back) the interactions from the logs in our database. We created screen recordings of the simulations of both participants' tablets and included them into VCode (Figure 5). This helped us to fully understand what was going on during the experiments, as VCode allows operating multiple videos simultaneously with only one playback controller.

To analyze participants' awareness of each other's activities while working on the task, we initially attempted to use the awareness indices identified by Hornecker et al. [9]. However, these indices were not designed for a multi-display environment. Therefore interactions with the personal displays between participants were not incorporated. For instance, they did not comprise situations where participants used 
their tablet to show a document to their partner without communicating. We therefore analyzed the video recordings regarding interaction patterns and task solving strategies used by the participants to solve the given task. For each participant group and condition we rated various factors including the closeness of collaboration, the amount of interactions on the tablets, the amount of parallel vs. complementary work and the amount of reading and rechecking grouped elements, to identify collaborative strategies in a setup with personal tablets as additional tools.

One-way repeated measures analysis of variance (RM-ANOVA) tests were conducted to analyze statistical differences between the base condition and the condition with the tablets regarding task performance (task solution time) and efficiency (e.g. number of interactions). Bonferroni correction was applied when comparing individual conditions. Further analysis of variance tests were conducted to find differences between the different transfer techniques. Friedman tests were used for analyzing 5-point Likert-scale ratings from post-experiment questionnaires and Wilcoxon Signed-Rank Tests with Bonferroni correction (significance level of 0.017) as post-hoc tests for comparing differences between pairs of conditions. For all other tests, a significance level of 0.05 was used.

\section{Results and Discussion}

\subsection{Task Solving Strategies}

During the study we observed how participant pairs solved the sorting task and their use of the shared and personal displays. As there was no best way to find the solution, strategies varied between groups but also between conditions with different transfer techniques. All pairs collaborated close together in the conditions without tablets, except one team that worked parallel for short periods. In our interview one participant said that the condition without tablets "forced them to collaborate together". Therefore the tabletop-only condition is not discussed in this section. Adding the personal displays allowed participants to choose diverse strategies. A common approach was to start with finding the first and last page of the chapter followed by looking for subsequent and/or precedent pages depending on the approach. Whereas the first page was placed either in the top or bottom left side of the shared surface, the last page was usually placed on the right side and represented the frame for the other documents.

The handling of the documents on the shared surface differed as well and was dependent on the used tactic. When a lot of documents were enlarged, more time was used for the arrangement of documents on the shared surface. Some teams did not enlarge documents at all and just used the tablets for reading. We calculated a scale factor of documents to determine their size over time. In the conditions with tablets, the documents' sizes over time (between 0.173 and 0.177 ) were even less than half of the sizes in conditions without tablets $(0.371)$.

Within the conditions with the tablets, we identified four different strategies: The first strategy (team-up) is characterized by close collaboration throughout the task. The second strategy (split-up) is the exact opposite resulting in parallel work 
throughout the task. The third strategy (prepare-and-conquer) is separated into an initial phase of parallel work followed by close collaboration towards the end of the task. The fourth strategy (divide-and-combine) is characterized by recurring changes between close and loose collaboration. The four strategies are described in detail below, including examples of participants' tactics.

Team-Up. The first strategy was picked in twelve out of the 24 trials with tablets. It was characterized by very close collaboration between participants throughout most parts of the task. Participants did not spend time working on parallel activities (e.g. trying to find subsequent pages on their own) but teamed up to find relations between pages or even a specific page. Within this strategy, various tactics emerged. For instance, in the beginning participants opened five different documents on each tablet and started to search for relations between pages using the personal displays only. Other groups enlarged one document on the shared surface and tried to find subsequent pages on their tablets. However, this tactic of opening five documents on each tablet did not necessarily entail close collaboration. Other groups used this tactic to split up and work on their own.

Within the team-up strategy another tactic consisted of opening the same documents on both tablets to discuss relations between pages. Another approach was to work on the shared surface using the personal displays just occasionally. In this tactic the tablets were often positioned on the horizontal space on the shorter sides of the table and picked up for use when needed.

Split-Up. The second strategy involved splitting up of the task and working on separate activities in parallel resulting in loose collaboration. When splitting up, participants focused on reading on their tablets on their own, or browsed for pages on the shared surface without communicating or interacting with their partner. Only in five out of the 24 trials this strategy was chosen by three of eight teams. We expected pairs with participants who did not know each other from before to work parallel, but one of these two teams worked surprisingly close together. On the contrary, a couple decided to split up and both participants tried to find matching pages on their own.

Prepare-and-Conquer. In the third strategy partners began to work on parallel activities (split-up) during the initial phase of the task. In this period, participants tried to gain an overview of the chapter or just of a number of documents. Communication was reduced to be able to concentrate on reading. After participants found the first pages that belonged together, they switched to close collaboration (team-up) and worked together to complete the task as a team. The name of this strategy was determined by the initial "preparation" phase that helped "conquer" the task afterwards. This strategy was only chosen in four out of the 24 trials by three of the eight teams.

Divide-and-Combine. The fourth strategy was characterized by recurring changes in the amount of close collaboration. Teams regularly evaluated and changed their strategies based on the current situation. The name of this strategy in based on its pattern: Participants "divided" the task into parallel activities when necessary and "combined" their results repeatedly. For instance, after both participants arranged some documents on the shared surface and found some matching pages on the 
tabletop, they changed to parallel work with both participants searching for one specific document. When working in parallel, participants often spent time reading on the tablets. The divide-and-combine strategy was also found when participants were working on the shared surface only. However, only three out of the 24 trials with tablets (by three of the eight teams) were explicitly rated as divide-and-combine strategy. When we could not find separation of tasks but only close collaboration, trials were rated as team-up strategy.

We compared task completion times of all team-up strategies with the conjunction of times of all others strategies. On average, teams that used the team-up strategy with close collaboration were faster than teams choosing one of the other three strategies with parallel work included. Task completion times of team-up strategies were only slightly longer compared to the experiments without the use of tablets.

\subsection{Transfer Techniques and Task Performance}

Errors. All eight participant pairs were successful in completing the sorting task. Only in eight of the 32 trials (eight groups, four conditions) six groups made mistakes in the final sorting of the pages, resulting in 32 wrong (out of 288) pairings overall. We could not find any significant differences between conditions regarding the error points. We further could not find any significant differences between the four book chapters regarding errors.

Time. The mean task completion time per trial was 11 minutes and 28 seconds (SD: 3 minutes 48 seconds). We could not find any significant differences between the four book chapters regarding solution times.

We assumed that the more complicated the transfer technique was, the longer participants would need to solve the task. However, our data showed the opposite behavior (Figure 6). Task performance measurements showed that the buttons technique was slower compared to the others. Nevertheless, participants rated the buttons technique as the easiest. The lenses technique was reported to be more complicated and required too many steps, but was the fastest. The times differed significantly across the conditions $(\mathrm{F}(3,21)=4.488, \mathrm{p}=0.014)$, but only the in buttons condition times were significantly higher $(\mathrm{M}=14.429$, 95\% CI [10.62, 18.24]) compared to the lenses condition $(\mathrm{M}=9.783,95 \% \mathrm{CI}[7.96,11.61])$ with $\mathrm{p}=$ 0.02 .

We investigated the results and searched for explanations with the amount of use of the tablets. Some groups used the tablets less in the lenses condition compared to the other two conditions. Yet conditions with less use of the tablets did not necessarily finish faster than other groups. The analysis of interactions per minute on the tablets showed significant differences $(\mathrm{F}(2,14)=4.610, \mathrm{p}=0.029)$ between conditions. However, we could not find significant differences when comparing the conditions pairwise. Comparing the interactions per minute on the shared surface resulted in significant differences $(\mathrm{F}(3,21)=6.980, \mathrm{p}=0.002)$ between the condition without tablets $(\mathrm{M}=62.506,95 \% \mathrm{CI}[43.68,81,33])$ and the buttons conditions $(\mathrm{M}=35.301$, $95 \%$ CI $[22.69,47.92])$ with $\mathrm{p}=0.025$. 


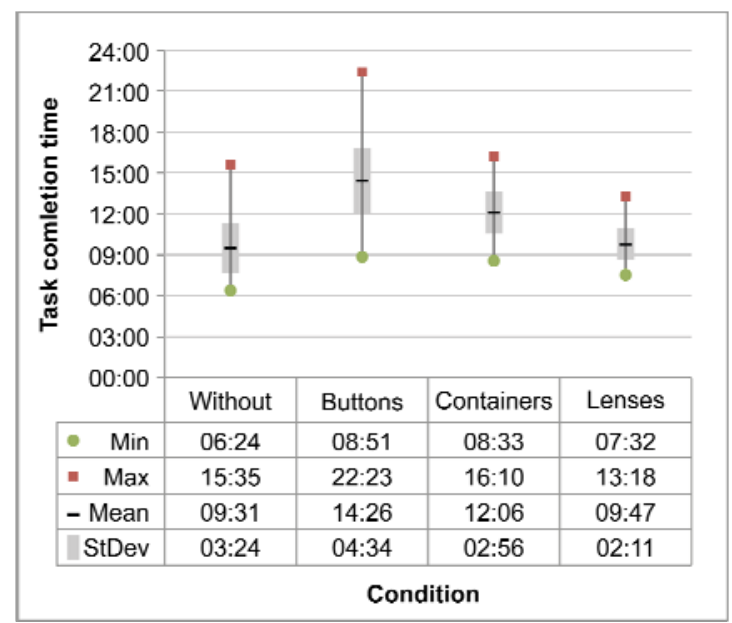

Fig. 6. Minimum, maximum and mean task completion times for the four conditions including standard deviation

With the help of the video analysis we discovered that participants showed confused and uncontrolled behavior in the buttons and containers conditions. The analysis of the number of document transfers per minute from the shared to the personal displays revealed significant differences $(\mathrm{F}(2,14)=11.966, \mathrm{p}=0.001)$ between the buttons condition $(\mathrm{M}=3.641,95 \% \mathrm{CI}[1.99,5.3])$ and the containers condition $(\mathrm{M}=1.89,95 \% \mathrm{CI}[0.77,3.01])$ with $\mathrm{p}=0.043$ and between the buttons condition and the lenses condition $(\mathrm{M}=1.135,95 \% \mathrm{CI}[0.52,1.75])$ with $\mathrm{p}=0.011$. Participants often closed and reopened documents repetitively to check their contents. The costs (in terms of number of touches and completion time) for transferring a document to the tablet is higher the more complicated a transfer technique is. Therefore, in the lenses technique participants seemed to reflect more about which documents they wanted to examine and which documents had already been reviewed or currently had been excluded from investigation.

Qualitative Findings. The subjective rating of participants after the experiment showed clear preference of the buttons technique, followed by containers and lenses techniques. Reasons retrieved from interviews included the fast and easy use of the buttons beneath the documents. Additionally, participants could easily send documents to their partners, which was especially helpful when one participant wanted to send documents to both tablets. Advantages of the containers technique included the behavior that reminded users of the use of desktop computers and that the user feedback that was better compared to the other techniques. One participant noted that dragging the documents into the containers was time consuming. The lenses technique was rated as most complicated and not efficient. A reason was that a series of steps had to be followed to transfer documents. In contrast, one participant said that after he figured out how to use it, the transfer was surprisingly easy. Two participants rated the lenses technique as their favorite as it was fun and engaging to 
use. One participant claimed that having to move the tablet across the surface even increased the awareness of the participant's activity in the lenses technique.

The complexity of the lenses technique can be traced back to our workaround with the fiduciary marker that required additional steps for transferring the documents. Compared to the buttons and containers techniques that only required one interaction to transfer a document, the lenses technique required four: Pressing the button, moving the tablet across the surface, releasing the button and finally selecting the document(s) to open. Improving the detection of the tablet's position could potentially reduce the transfer to two steps.

Another difference between the techniques is the metaphor of transferring documents resulting in the separation of interactions. Whereas in the buttons and containers techniques users have to transfer documents from the shared display by touching the corresponding buttons or dragging them into containers, the lenses technique allows grabbing documents from the shared surface without interactions on the shared surface. The metaphor of "taking a photo", like one participant noted, also impacts the task itself. The personal displays can be used for examining the documents. The shared display acts as the link between participants, representing their progress, for arrangement of documents and for building groups of pages that belong together in order to solve the task. Saving time for scaling and arrangement of documents on the shared surface combined with considerate opening of specific documents and reading them on the tablets was one efficient strategy to solve the task.

\subsection{Awareness}

For identifying differences between conditions relating to awareness we analyzed qualitative self-assessments from participants from the post-experiment questionnaire. Although the awareness in the condition without tablets was rated slightly higher and in the lenses condition slightly lower, we could not find any significant differences $\left(\chi^{2}(3)=1.966, \mathrm{p}=0.58\right)$. Results from our interviews were overall balanced despite contradictory opinions about the perceived awareness.

One participant said: "When using the tablets it is harder to communicate and you do not know what the other one is reading". Other participants stated that using the tablets resulted in higher awareness. When comparing the results to the subjective ratings of participants to our assigned strategies, teams with close collaboration (team-up) rated high awareness in the conditions with the tablets. Another participant noted that "the tabletop gives a feeling of connectedness and what is going on". Since documents had to be grouped on the shared surface, it acted like a progress view for the task. The color-coding we used for highlighting opened documents on the shared surface helped participants to maintain an awareness of collaborators' activities. For instance, one participant stated: "When I looked at the screen [shared surface] I knew what he was doing". He added that he could see what documents his partner had opened but not which exact document he was currently reading. Interestingly, one participant claimed that she was not aware what her collaborator was exactly doing in the condition without the tablets. She explained this with saying that the shared surface was messier because of all the enlarged documents on it, thus making it more difficult to maintain an overview. 


\section{Conclusion}

Our study is the first to analyze a collaborative MDE with multi-touch input on both shared and personal displays. To investigate the effects of multi-touch personal devices on task performance, collaboration strategies and awareness in multi-display environments we proposed three different techniques for one-way transfer of documents from a shared surface to tablets serving as personal devices. In the first technique (buttons), each document featured a button for transferring the documents. In the second technique (containers), the shared surface featured two containers representing the participants' tablets. The third technique (lenses) allowed participants to pick up documents by moving the tablet above the tabletop. The three main contributions of this paper are:

First, the identification of four different strategies teams used to coordinate and collaborate, ranging from close collaboration to parallel work throughout the task. The identified collaboration strategies are specific to MDEs. Teams varied strategies between the conditions with different transfer techniques. These findings extend the importance of transient design [10] to allow users to follow specific strategies for multi-display environments. Adding personal displays to collaborative environments further allows for completing tasks on either display, therefore adding to the flexibility of the environment. In the condition without the additional tablets, some groups felt forced to close collaboration, which did not result in significant faster task completion times.

Second, the indicative assessment of users' performance with respect to three oneway transfer techniques in a multi-touch based MDE. The analysis of task completion times showed that the lenses technique was significantly faster compared to the buttons technique. This result could be attributed to the fact that the simplicity of the buttons technique led to an increased document transfer between devices and less coordinated task-solving strategies. Ratings and qualitative feedback from participants indicated that the sequence of interactions required to transfer documents with the lenses technique were too complex, which can be attributed to technical limitations of our implementation. Using an improved mechanism for recognizing and tracking tablets would eliminate the use of a fiduciary marker, simplifying the interaction. The costs for transferring documents from the shared surface onto the tablets (in terms of interactions) seemed to not only have an impact on task-solving strategies and coordination, but was also an important factor for participants when comparing transfer techniques. Further research could investigate the effect of costs on tasksolving strategies. More research is also needed to analyze the suitability of the techniques for different scenarios by comparing task types.

Third, the assessment of users' awareness of their collaborator's activities in a multi-touch based MDE, which showed no significant differences between the conditions, although we expected the condition with the shared surface only to receive higher rating in awareness. Qualitative participant feedback showed that using the shared surfaces as task progress view and for providing feedback about activities taking place on the personal displays is important for maintaining awareness amongst users. 


\section{References}

1. Community Core Vision, http: / / ccv . nuigroup. com

2. Echtler, F., Nestler, S., Dippon, A., Klinker, G.: Supporting casual interactions between board games on public tabletop displays and mobile devices. Pers. Ubiquit. Comput. 13, 609-617 (2009)

3. Forlines, C., Wigdor, D., Shen, C., Balakrishnan, R.: Direct-touch vs. mouse input for tabletop displays. In: SIGCHI Conference on Human Factors in Computing Systems, pp. 647-656. ACM, San Jose (2007)

4. Fox, A., Johanson, B., Hanrahan, P., Winograd, T.: Integrating information appliances into an interactive workspace. In: Computer Graphics and Applications, pp. 54-65. IEEE, Los Alamitos (2000)

5. Free eBooks by Project Gutenberg, http: / / www . gutenberg . org

6. Ha, V., Inkpen, K.M., Whalen, T., Mandryk, R.L.: Direct Intentions: The Effects of Input Devices on Collaboration around a Tabletop Display. In: 1st IEEE International Workshop on Horizontal Interactive Human-Computer Systems, pp. 177-184. IEEE Computer Society, Washington (2006)

7. Hagedorn, J., Hailpern, J., Karahalios, K.G.: VCode and VData: illustrating a new framework for supporting the video annotation workflow. In: Working Conference on Advanced Visual Interfaces, pp. 317-321. ACM, New York (2008)

8. Hawkey, K., Kellar, M., Reilly, D., Whalen, T., Inkpen, K.M.: The proximity factor: impact of distance on co-located collaboration. In: ACM SIGGROUP Conference on Supporting Group Work, pp. 31-40. ACM, Sanibel Island (2005)

9. Hornecker, E., Marshall, P., Dalton, N.S., Rogers, Y.: Collaboration and interference: awareness with mice or touch input. In: ACM Conference on Computer Supported Cooperative Work, pp. 167-176. ACM, San Diego (2008)

10. Isenberg, P., Fisher, D., Morris, M., Inkpen, K., Czerwinski, M.: An exploratory study of co-located collaborative visual analytics around a tabletop display. In: IEEE Symposium on Visual Analytics Science and Technology (VAST), pp. 179-186 (2010)

11. Kaltenbrunner, M., Bencina, R.: reacTIVision: a computer-vision framework for tablebased tangible interaction. In: 1st International Conference on Tangible and Embedded Interaction, pp. 69-74. ACM, New York (2007)

12. Kin, K., Agrawala, M., DeRose, T.: Determining the benefits of direct-touch, bimanual, and multifinger input on a multitouch workstation. In: Graphics Interface 2009, pp. 119124. Canadian Information Processing Society, Kelowna (2009)

13. MT4J - Multitouch for Java, http: / / www . mt $4 \mathrm{j}$. org

14. Müller-Tomfelde, C., Schremmer, C.: Touchers and mousers: commonalities and differences in co-located collaboration with multiple input devices. In: 26th Annual SIGCHI Conference on Human Factors in Computing Systems, pp. 1149-1152. ACM, Florence (2008)

15. Müller-Tomfelde, C., Wessels, A., Schremmer, C.: Tilted tabletops: In between horizontal and vertical workspaces. In: 3rd IEEE International Workshop on, Horizontal Interactive Human Computer Systems, TABLETOP 2008, pp. 49-56 (2008)

16. Nacenta, M.A., Gutwin, C., Aliakseyeu, D., Subramanian, S.: There and back again: crossdisplay object movement in multi-display environments. Human-Computer Interaction 24, 170-229 (2009)

17. Nacenta, M.A., Aliakseyeu, D., Subramanian, S., Gutwin, C.: A comparison of techniques for multi-display reaching. In: SIGCHI Conference on Human Factors in Computing Systems, pp. 371-380. ACM, Portland (2005) 
18. North, C., Dwyer, T., Lee, B., Fisher, D., Isenberg, P., Robertson, G., Inkpen, K.: Understanding multi-touch manipulation for surface computing. In: Gross, T., Gulliksen, J., Kotzé, P., Oestreicher, L., Palanque, P., Prates, R.O., Winckler, M. (eds.) INTERACT 2009. LNCS, vol. 5727, pp. 236-249. Springer, Heidelberg (2009)

19. Olwal, A., Feiner, S.: Spatially aware handhelds for high-precision tangible interaction with large displays. In: 3rd International Conference on Tangible and Embedded Interaction, pp. 181-188. ACM, Cambridge (2009)

20. Plaue, C., Stasko, J., Baloga, M.: The conference room as a toolbox: technological and social routines in corporate meeting spaces. In: 4th International Conference on Communities and Technologies, pp. 95-104. ACM, University Park (2009)

21. Rekimoto, J.: Pick-and-drop: a direct manipulation technique for multiple computer environments. In: 10th Annual ACM Symposium on User Interface Software and Technology, pp. 31-39. ACM, New York (1997)

22. Rogers, Y., Lindley, S.: Collaborating around vertical and horizontal large interactive displays: which way is best? Interacting with Computers 16, 1133-1152 (2004)

23. Scott, S.D., Grant, K.D., Mandryk, R.L.: System guidelines for co-located, collaborative work on a tabletop display. In: 8th conference on European Conference on Computer Supported Cooperative Work, pp. 159-178. Kluwer Academic Publishers, Helsinki (2003)

24. Scott, S.D., Sheelagh, M., Carpendale, T., Inkpen, K.M.: Territoriality in collaborative tabletop workspaces. In: ACM Conference on Computer Supported Cooperative Work, pp. 294-303. ACM, Chicago (2004)

25. Shen, C., Everitt, K., Ryall, K.: UbiTable: Impromptu Face-to-Face Collaboration on Horizontal Interactive Surfaces. In: Dey, A.K., Schmidt, A., McCarthy, J.F. (eds.) UbiComp 2003. LNCS, vol. 2864, pp. 281-288. Springer, Heidelberg (2003)

26. Streitz, N.A., Geißler, J., Holmer, T., Konomi, S., Müller-Tomfelde, C., Reischl, W., Rexroth, P., Seitz, P., Steinmetz, R.: i-LAND: an interactive landscape for creativity and innovation. In: SIGCHI Conference on Human Factors in Computing Systems, pp. 120127. ACM, New York (1999)

27. Streitz, N., Prante, T., Müller-Tomfelde, C., Tandler, P., Magerkurth, C.: Roomware@: the second generation. In: CHI 2002 Extended Abstracts on Human Factors in Computing Systems, pp. 506-507. ACM, Minneapolis (2002)

28. Tan, D., Gergle, D., Mandryk, R., Inkpen, K., Kellar, M., Hawkey, K., Czerwinski, M.: Using job-shop scheduling tasks for evaluating collocated collaboration. Pers. Ubiquit. Comput. 12, 255-267 (2008)

29. Tang, A., Tory, M., Po, B., Neumann, P., Carpendale, S.: Collaborative coupling over tabletop displays. In: SIGCHI Conference on Human Factors in Computing Systems, pp. 1181-1190. ACM, Montréal (2006)

30. Tse, E., Shen, C., Greenberg, S., Forlines, C.: How pairs interact over a multimodal digital table. In: SIGCHI Conference on Human Factors in Computing Systems, pp. 215-218. ACM, San Jose (2007)

31. Wallace, J.R., Scott, S.D., Stutz, T., Enns, T., Inkpen, K.: Investigating teamwork and taskwork in single- and multi-display groupware systems. Pers. Ubiquit. Comput. 13, 569581 (2009)

32. Wilson, A.D., Benko, H.: Combining multiple depth cameras and projectors for interactions on, above and between surfaces. In: 23nd Annual ACM Symposium on User Interface Software and Technology, pp. 273-282. ACM, New York (2010)

33. Wu, M., Balakrishnan, R.: Multi-finger and whole hand gestural interaction techniques for multi-user tabletop displays. In: 16th Annual ACM Symposium on User Interface Software and Technology, pp. 193-202. ACM, New York (2003) 